\title{
A Broadband Corrugated Horn for a W-band Gyro-TWA
}

\author{
Paul McElhinney, Craig R. Donaldson, Wenlong He, Liang Zhang, Kevin Ronald, \\ Alan D. R. Phelps and Adrian W. Cross \\ SUPA, Department of Physics, University of Strathclyde, Glasgow, G4 ONG, UK
}

\begin{abstract}
A quasi-optical mode converter in the form of a corrugated horn has been constructed for a W-band gyrotron traveling wave amplifier (gyro-TWA). The prototype was designed and optimized through analytical and numerical simulations. The horn converts a cylindrical $T E_{11}$ mode into a free-space TEM ${ }_{00}$ mode in a frequency band of $90 \mathrm{GHz}$ to $100 \mathrm{GHz}$. The optimized simulation predicts a return loss better than $-35 d B$ and a Gaussian coupling efficiency of $97.8 \%$ and the measured prototype demonstrates a close agreement with these figures.
\end{abstract}

Keywords: Corrugated horn; Gyro-TWA; quasi-optical.

\section{Introduction}

There is a growing need for the development of high power coherent microwave sources at shorter wavelengths $(\mathrm{mm}$ and sub-mm), especially in the fields of plasma physics [1], remote sensing and imaging [2] and for electron spin resonance spectroscopy [3]. Gyro-devices are by their nature particularly suited to this type of application due to the fast-wave cyclotron resonance maser instability, which is capable of producing high power radiation at frequencies that prove challenging for other sources.

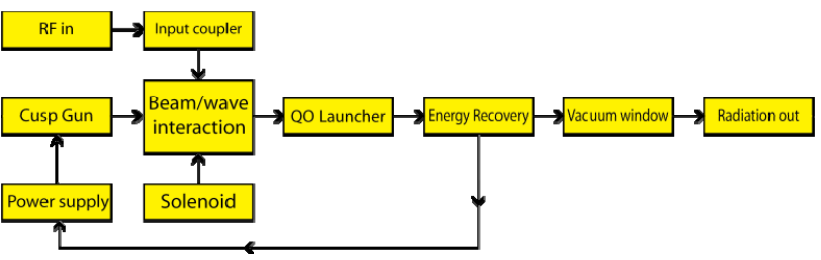

Fig. 1 System design scheme for a gyro-TWA

A W-band gyro-TWA $[4,5]$ based on a cusp electron beam source [6-8] and a helically corrugated interaction region [9-11] is currently under development to provide a continuously tunable source over the range between 90 $\mathrm{GHz}$ to $100 \mathrm{GHz}$ with a CW power output of $\sim 10 \mathrm{~kW}$. Fig 1 gives an overview of the system design: The cusp electron beam source is capable of producing an axis encircling annular electron beam at $40 \mathrm{kV}$ and $1.5 \mathrm{~A}$ with an adjustable beam alpha up to 3. Radiation is then produced in the helically corrugated interaction region due to the coupling of the beam with the operating eigenwave, which is the result of the superposition of the co-rotating $\mathrm{TE}_{11}$ and the counter-rotating $\mathrm{TE}_{21}$ mode. This design allows for an increase in the overall bandwidth while maintaining high efficiency by modifying the coupling relationship between the beam and the eigenwave $[12,13]$.

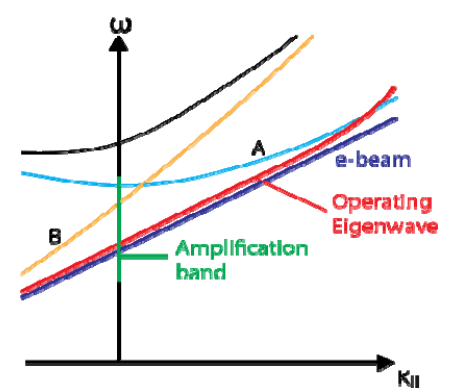

Fig. 2 Operational eigenmode for helical interaction region

The electron beam is then decoupled from the radiation by means of a quasi-optical mode converter that converts the fundamental operating mode within the gyro-TWA $\left(\mathrm{TE}_{11}\right)$ to a hybrid mode, which is generally accepted to consist of $85 \% \mathrm{TE}_{11}$ and $15 \% \mathrm{TM}_{11}$ (by power) and is closely coupled to the fundamental free space Gaussian mode $\left(\mathrm{TEM}_{00}\right)$. This Gaussian radiation beam may then pass through the collector system and vacuum window unperturbed while the electrons are collected at the energy recovery system that is predicted to increase overall efficiency to $40 \%$ by recovering the energy of the spent electron beam [14-16]. This type of corrugated mode converting horn was chosen over more conventional beam-wave decoupling methods due to the perceived performance advantages that it makes possible; both greater bandwidth and the capability to provide a source that is continuously tunable over this bandwidth. From an initial the primary consideration was the reduction of the reflection, which was best achieved using a $\sin ^{2}$ profile as described by Clarricoats and Olver [17]. From this baseline design a prototype model was constructed with corrugations linearly tapered in depth from $\lambda / 2$ at the throat of the horn to $\lambda / 4$ at the aperture, to produce optimum conditions for impedance matching between the horn and the gyro-TWA and minimize the reflection. The corrugation and vane length were initially set to $\lambda / 10$ and $\lambda / 30$. This prototype design was then numerically optimized over the operating frequency band. This was done using a mode matching technique, which allows for fast implementation and optimization of various designs and geometries and a high degree of freedom when selecting the design parameters.

\section{Results}

The final optimized results can be seen in Fig. 3. The yellow (dotted line) is the normalized power of the $\mathrm{TE}_{11}$ mode and the purple (dot-dash) line is the normalized 
power of the $\mathrm{TM}_{11}$ mode. It can be seen that both are close to the desired values of $85 \%$ and $15 \%$ respectively. There is slightly less power in the $\mathrm{TM}_{11}$ mode due to the inclusion of higher order modes, which is an unavoidable consequence of the output aperture dimensions. After this iteration process was concluded and the final optimized model was manually "tuned" the result is a far-field radiation pattern and aperture field that corresponds with a high directivity Gaussian beam that has more than $99.9 \%$ of the power contained within an angle of less than $19^{\circ}$ and a return loss of better than $-35 \mathrm{~dB}$. The Gaussian coupling coefficient was calculated to be 0.978 and the cross-polar component of the radiation pattern was found to be less than $-40 \mathrm{~dB}$.

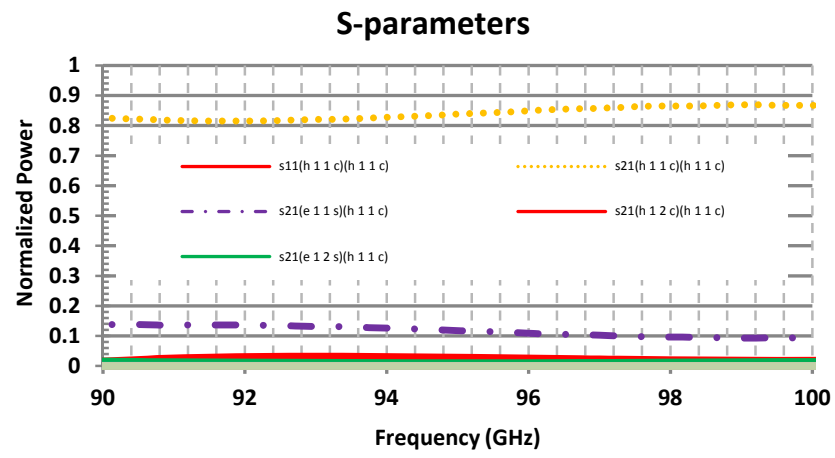

Fig. 3 Mode percentage (power) (legend real difficult to read)

The prototype horn was constructed using an aluminum former that was machined in-house on a computer controlled lathe. Copper was then grown onto the former and the aluminum was dissolved. The performance of this prototype was measured on a W-band vector network analyzer and the reflection and far field characteristics were determined over the frequency range between 90 and 100 $\mathrm{GHz}$. The measured performance of the prototype horn was very similar to the simulated prediction. The $\mathrm{S}_{11}$ performance was less than $-30 \mathrm{~dB}$ over the measured bandwidth and the width of the primary lobe was better than the simulated result.

\section{References}

[1] T. Imai, et al., "ITER R\&D: Auxiliary systems: Electron cyclotron heating and current drive system", Fusion Eng. Des., 55(2-3), pp.281-289, 2001.

[2] W. M. Manheimer, G. Mesyats, and M. I. Petelin, pp.169-207 in Applications of High-Power Microwaves, A. V. Gaponov-Grekhov and V. L. Granatstein, Eds., Norwood, MA: Artech House, 1994.

[3] T. I. Smirnova, A. I. Smirnov, and R. B. Clarkson, "W-band (95 GHz) EPR spectroscopy of nitroxide radicals with complex proton hyperfine structure: Fast motion", J. Phys. Chem., 99 (22), pp. 9008-9016, 1995.
[4] G.G. Denisov et al., "Gyrotron traveling wave amplifier with a helical interaction waveguide", Phys. Rev. Lett., 81 (25), pp. 5680-5683, 1998.

[5] V. L. Bratman, et al., "High-gain wide-band gyrotron traveling wave amplifier with a helically corrugated waveguide", Phys. Rev. Lett., 84, (12), pp. 2746-2749, 2000.

[6] C. R. Donaldson, W. He, A.W. Cross, et al., "A cusp electron gun for millimeter wave gyrodevices," Appl. Phys. Lett., 96 (14), 141501, 2010.

[7] C. R. Donaldson, W. He, A. W. Cross, et al. "Design and Numerical Optimization of a Cusp-Gun-Based Electron Beam for Millimeter-Wave Gyro-Devices", IEEE Trans. Plasma Sci., 37 (11), pp. 2153-2157, 2009.

[8] W. He, et al., "Axis-encircling electron beam generation using a smooth magnetic cusp for gyrodevices", Appl. Phys. Lett., 93(12), 121501, 2008.

[9] G. Burt, et al., "Dispersion of helically corrugated waveguides: Analytical, numerical, and experimental study”, Phys. Rev. E, 70 (4), 046402, 2004.

[10] A. W. Cross, et al., "Helically corrugated waveguide gyrotron traveling wave amplifier using a thermionic cathode electron gun", Appl. Phys. Lett., 90 (25), 253501, 2007.

[11] W. He, et al., "Theory and simulations of a gyrotron backward wave oscillator using a helical interaction waveguide", Appl. Phys. Lett., 89 (9), 091504, 2006.

[12] W. He, et al., "Gyro-BWO experiments using a helical interaction waveguide", IEEE Trans. Electron Devices, 52 (5), pp. 839-844, 2005.

[13] W. He, et al., "W-band Gyro-devices Using Helically Corrugated Waveguide and Cusp Gun: Design, Simulation and Experiment", TST, 4 (1), pp. 9-19, 2011.

[14] L. Zhang, et al., "Design of an Energy Recovery System for a Gyrotron Backward-Wave Oscillator", IEEE Trans. Plasma Sci., 37 (3), pp. 390-394, 2009.

[15] L. Zhang, et al., "Numerical Optimization of a Multistage Depressed Collector With Secondary Electron Emission for an X-band Gyro-BWO", IEEE Trans. Plasma Sci., 37 (12), pp. 2328-2334, 2009.

[16] L. Zhang, et a., "W-band gyro-BWO with a four stage depressed collector," TST, 4 (3), pp. 76-84, (2011).

[17] P. J. B. Clarricoats and A. D. Olver, "Corrugated Horns for Microwave Antennas", Peter Peregrinus: London, 1984. 